\title{
Digital Photography Study File
}

National Cancer Institute

\section{Source}

National Cancer Institute. Digital Photography Study File. NCI Thesaurus. Code C115500.

A collection of digital photographs pertaining to a clinical study. 